\title{
Effects of feeding monensin to bred heifers fed in a drylot on nutrient and energy balance
}

\author{
Courtney N. Hemphill,* Tryon A. Wickersham,* Jason E. Sawyer,* \\ T. M. Brown-Brandl ${ }^{\dagger}$, Harvey C. Freetly, ${ }^{\dagger}$ and Kristin E. Hales ${ }^{\dagger, 1}$ \\ *Department of Animal Science, Texas A\&M University, College Station, TX 77843; and 'USDA, ARS, U.S. \\ Meat Animal Research Center, Clay Center, NE 68933
}

\begin{abstract}
The objective of this study was to determine if feeding monensin would improve diet digestion, energy and nitrogen balance in bred heifers receiving a limit-fed corn stalk-based diet. Sixteen pregnant Meat Animal Research Center (MARC) III composite heifers were used in a 161-d completely randomized design. Heifers were randomly assigned to one of two treatments, no monensin (CON) or $150 \mathrm{mg} / \mathrm{d}$ monensin (MON), with eight heifers in each treatment group. Heifers were limit-fed a corn stalk-based diet at $100 \%$ of $\mathrm{ME}_{\mathrm{m}}$ requirements. Effects of monensin on energy and nitrogen balance were determined via total fecal and urine collections and open-circuit respiration calorimetry. Total fecal and urine collection occurred on d 14, 42, and 161 of monensin feeding, and calorimetry measurements were made on d $0,3,14,28,42$, and 161 of monensin feeding. DMI was not different $(P=0.94)$ for CON and MON heifers and, by design, increased $(P<0.01)$ from d 14 to $\mathrm{d} 161$ of the trial to account for
\end{abstract}

increasing fetal growth requirements. No differences $(P=0.91)$ in GE intake were observed between CON and MON heifers, and DE and ME intakes did not differ $(P>0.58)$ with monensin inclusion. DM, OM, NDF, and ADF digestion did not differ $(P>0.52)$ between treatments. Fecal, methane, urinary, and heat energy losses were not different $(P>0.16)$ for MON and CON heifers. Methane production was not different between treatments when expressed as daily liters of methane $(P=0.40)$; however, $\mathrm{MON}$ heifers produced $7 \%$ less $(P=0.03)$ methane per day than $\mathrm{CON}$ heifers when expressed as liters of methane produced on a metabolic body weight (MBW) basis. Furthermore, monensin had no effect $(P=0.36)$ on overall retained energy (RE). Nitrogen intake and excretion was not different $(P>0.13)$ between treatment groups. Results of this experiment indicate that adding monensin to limit-fed, corn stalk-based diets may not have a large effect on the energy and nitrogen balance of confined heifers.

Key words: energy balance, heifers, limit feeding, monensin

Published by Oxford University Press on behalf of American Society of Animal Science 2018. This work is written by (a) US Government employees $(s)$ and is in the public domain in the US.

J. Anim. Sci. 2018.96:1171-1180 doi: 10.1093/jas/skx030

\section{INTRODUCTION}

As global population rises and land resources are diminished, innovative approaches to livestock production are required to meet global demand for food. One such strategy is the intensification of cow-calf systems, which involves

${ }^{1}$ Corresponding author: Kristin.Hales@ars.usda.gov

Received 18 October 2017.

Accepted 5 March 2018. limit feeding beef cows in drylots, similar to traditional feedlots. Intensified cow-calf systems give producers the flexibility to continue feeding cattle during times of limited forage availability rather than liquidating the cow herd (Eng, 2014). Furthermore, limit feeding has been reported to decrease energy requirements and improve feed utilization in beef cattle (Freetly and Nienaber, 1998; Trubenbach, 2014) and thus, provides a reasonable feeding strategy for intensified systems. 
Monensin is commonly included in traditional feedlot diets; however, its efficacy in limit-fed beef cows has not been studied extensively. Research conducted in beef steers has shown that monensin decreases the ruminal acetate to propionate ratio (Potter et al., 1976; Richardson et al., 1976; Thornton and Owens, 1981), reduces methanogenesis (Dinius et al., 1976; Thornton and Owens, 1981; Wedegaertner and Johnson, 1983), and improves feed efficiency by decreasing DMI and increasing ADG (Potter et al., 1976; Raun et al., 1976; Duffield et al., 2012).

Monensin inclusion in limit-fed diets may be a valuable additional management strategy for further enhancing the efficiency of feed utilization in limit feeding systems. Objectives of the current study were to determine if feeding monensin would increase retained energy (RE) and nitrogen balance of bred heifers receiving limit-fed corn stalk-based diets.

\section{MATERIALS AND METHODS}

This research was conducted according to experimental protocols approved by the U.S. Meat Animal Research Center (MARC) Institutional Animal Care and Use Committee. Sixteen pregnant MARC III (1/4 Angus, 1/4 Hereford, 1/4 Red Poll, $1 / 4$ Pinzgauer; initial BW $483 \pm 7.7 \mathrm{~kg}$ ) were used in a 161-d completely randomized design to determine the effects of feeding monensin on energy balance and nutrient utilization when heifers were limit-fed a corn stalk-based diet. Heifers were stratified by BW to one of two treatments, $150 \mathrm{mg}$ of monensin per day (MON) or no monensin (CON), with eight heifers in each treatment group. A lower concentration of monensin (150 mg/d vs. $300 \mathrm{mg} / \mathrm{d})$ was chosen as the authors speculated that producers may feed monensin at the lower concentration in a drylot situation when limit-feeding replacement heifers or cows. Heifers were housed in a semi-enclosed barn open to the south that was fitted with a Calan gate feeding system (American Calan, Northwood, NJ). Heifers had continuous access to fresh water and were fed individually. Diets were fed once daily at 0800 and consisted of corn stalks, corn silage, and wet distillers grains with solubles (Tables 1 and 2). Monensin was delivered as a pelleted supplement that was top-dressed at $3 \%$ of the diet. Pellets of the same formulation, but not containing monensin were fed to $\mathrm{CON}$ at the same rate. Heifer intake was limited (below ad libitum consumption) to achieve $100 \%$ of estimated $\mathrm{ME}_{\mathrm{m}}$ requirements, with daily feed amounts recalculated for the first, second, and third trimester of gestation to account for fetal growth requirements. Prior to the start of the trial, heifers were adapted to close human contact, metabolism stanchions, fecal bags and harnesses, and headbox calorimeters to facilitate collection procedures.

Heifers were weighed individually and moved into individual metabolism stanchions $(87 \mathrm{~cm} \times$ $214 \mathrm{~cm}$ ) in an enclosed barn at the beginning of each collection period. Total fecal and urine collections were conducted over $96 \mathrm{~h}$ and occurred on $\mathrm{d}$ 14,42 , and 161 of feeding monensin. Total urine was collected by inserting a 24-French Foley catheter with a 75-mL balloon (Bardex, Murray Hill, NJ) into each heifer's bladder using a stylus. The balloon was inflated using $50 \mathrm{~mL}$ of sterile physiological saline. Tygon tubing was connected to the Foley catheter and terminated into a plastic carboy (18 liters) that contained $100 \mathrm{~mL}$ of $0.36 \mathrm{M} \mathrm{HCl}$ to prevent volatilization of $\mathrm{N}$. Canvas bags were

Table 1. Ingredient composition of corn stalk-based diets with or without $150 \mathrm{mg} / \mathrm{d}$ of monensin fed to drylot heifers at a maintenance level of intake

\begin{tabular}{lcc}
\hline \hline Ingredient & Control & Monensin \\
\hline Corn stalks, \% of DM & 80 & 80 \\
Corn silage, \% of DM & 10 & 10 \\
Wet distillers grains with solubles, & 7 & 7 \\
$\quad \%$ of DM & 3 & 0 \\
Supplement without monensin & \\
$\quad \%$ of DM & & 3 \\
Supplement with monensin & , \% of \\
$\quad$ DM & 0 & \\
\hline
\end{tabular}

${ }^{a}$ Supplement contained on a DM basis: $25.00 \% \mathrm{CP}, 3.17 \%$ ether extract, $14.49 \%$ calcium, $0.33 \%$ phosphorus, $3.99 \%$ salt, $1.64 \%$ sodium, $0.50 \%$ magnesium, $0.61 \%$ potassium, $0.26 \%$ sulfur, $41.86 \%$ ash, 0.0018 ppm cobalt, $0.4157 \%$ copper, $0.0042 \%$ iodine, $0.5164 \%$, $0.0907 \%$ manganese, $0.0008 \%$ selenium, $0.1449 \%$ zinc, $0.0334 \%$ thiamin, $0.0070 \%$ Vitamin A $(1,000,000 \mathrm{IU} / \mathrm{g})$, and $0.1050 \%$ Vitamin $\mathrm{E}(500 \mathrm{IU} / \mathrm{g})$.

${ }^{b}$ Supplement contained $700 \mathrm{~g} /$ ton of monensin.

Table 2. Nutrient composition of corn stalk-based diets with or without $150 \mathrm{mg} / \mathrm{d}$ of monensin fed to drylot heifers at a maintenance level of intake

\begin{tabular}{lcc}
\hline \hline Item & Control & Monensin $^{a}$ \\
\hline As-fed basis & 71.89 & 71.89 \\
DM, \% & & \\
DM basis & 83.19 & 83.19 \\
OM, \% & 66.81 & 66.81 \\
NDF, \% & 40.78 & 40.78 \\
ADF, \% & 8.63 & 8.63 \\
CP, \% & 0 & 150 \\
Monensin, mg & & \\
\hline
\end{tabular}

${ }^{a}$ The concentration of monensin was confirmed by laboratory analyses. 
placed on the heifers using a harness to collect total feces. A description of the harness was described by Tolleson and Erlinger (1989). Daily fecal and urine samples were weighed and a $3 \%$ subsample (wet basis) was collected and composited by heifer for each collection period. Feed refusals (when present) were collected and weighed each day and subsamples were composited by heifer.

Gas exchange was measured on d 0, 3, 14, 28, 42 , and 161 of feeding monensin using portable respiration calorimeters (headboxes) using open-circuit calorimetry. Heifers received their daily feed allowance inside the calorimeters and had access to fresh water inside the calorimeter throughout the 24-h gas collection period. Individual oxygen consumption, carbon dioxide production, and methane production were collected over $24 \mathrm{~h}$ on $\mathrm{d} 2$ of each collection period. Concentration of gases was determined as described by Nienaber and Maddy (1985) and heat production (HP) was calculated according to Brouwer (1965). At the end of the 96-h collection period, heifers were weighed and returned to their pens.

Diet, orts, and fecal samples were dried in a forced-air oven for $96 \mathrm{~h}$ at $55{ }^{\circ} \mathrm{C}$, allowed to air-equilibrate, and then weighed for determination of DM. Diet, orts, and fecal samples were then ground through a 1-mm screen using a Wiley mill (Thomas Scientific, Swedesboro, NJ) and dried at $105^{\circ} \mathrm{C}$ for $24 \mathrm{~h}$ for determination of DM. OM was determined as the loss in dry weight upon combustion in a muffle furnace for $8 \mathrm{~h}$ at $450{ }^{\circ} \mathrm{C}$. Analysis for NDF and ADF was performed sequentially using an Ankom Fiber Analyzer with sodium sulfite omitted and without correction for residual ash (Ankom Technology Corp., Macedon, NY).

Energy values for diet, ort, and fecal samples were determined by direct calorimetry using a Parr 6300 Calorimeter (Parr Instrument Co., Moline, IL). To analyze urinary energy, cotton rounds were weighed and placed into bomb calorimeter crucibles. Standards were created using the average energy content of the cotton rounds. Urine was added to the crucible $(4 \mathrm{~mL})$ and differences in energy content were attributed to the urine. The difference of the urine and standard was divided by the amount of urine added to determine GE. Diet, fecal, and urine samples were sent to a commercial laboratory (SDK Labs, Hutchinson, KS) for analysis of N. Mixed diet samples were also analyzed for monensin (Elanco Laboratory, Greenfield, IN).

All data were analyzed using the PROC MIXED procedure in SAS 9.3 (SAS Institute Inc., Cary, NC). Model fixed effects included treatment, day, and treatment $\times$ day. Day was a repeated term with heifer as the subject. Treatment means were calculated using the LSMEANS option, and the pdiff function was used to separate treatment means.

\section{RESULTS}

The diets used in the study were corn-stalk based and contained corn silage, wet distillers grains with solubles, and a commercial supplement (Table 1). The treatments differed in the presence of monensin in the commercial supplement. The diets were identical in NDF, ADF, CP, and OM (Table 2).

One heifer in the CON group died due to factors unrelated to treatment before the third collection period; thus, only seven $\mathrm{CON}$ heifers were sampled on d 161 of monensin feeding.

No treatment $\times$ day interactions were detected for any of the response variables tested $(P \geq 0.12)$. No differences in DMI (Table 3) were observed between $\mathrm{CON}$ and MON heifers $(P=0.94)$. By design, DMI increased from d 14 to d 161 to account for fetal growth requirements $(P<0.01)$. DM, OM, NDF, and ADF digestion (Table 3) did not differ between treatments $(P \geq 0.52)$. Digestion of $\mathrm{OM}$ was greatest on $\mathrm{d} 161(P<0.03)$, while NDF and ADF digestion were the lowest on d $42(P<0.02)$ and not different on $\mathrm{d} 14$ and $\mathrm{d} 161(P \geq 0.12)$.

Table 3. Diet and nutrient digestibility of drylot heifers receiving corn stalk-based diets with or without $150 \mathrm{mg} / \mathrm{d}$ of monensin at a maintenance level of intake

\begin{tabular}{|c|c|c|c|c|c|c|}
\hline \multirow[b]{2}{*}{ Item } & \multicolumn{3}{|c|}{ Day of study } & \multirow[b]{2}{*}{$\mathrm{SEM}^{e}$} & \multicolumn{2}{|c|}{ Probability $^{a}$} \\
\hline & $14^{b}$ & $42^{c}$ & $161^{d}$ & & Treatment & Day \\
\hline \multicolumn{7}{|c|}{ DM intake, g/d } \\
\hline Control & $6,010^{\mathrm{a}}$ & $5,302^{b}$ & $8,219^{\mathrm{c}}$ & 170.9 & 0.94 & $<0.01$ \\
\hline Monensin & $5,945^{\mathrm{a}}$ & $5,585^{b}$ & $8,039^{c}$ & 161.2 & & \\
\hline \multicolumn{7}{|c|}{ DM digestibility, \% } \\
\hline Control & 42.7 & 41.1 & 44.4 & 2.16 & 0.52 & 0.77 \\
\hline Monensin & 44.8 & 43.8 & 43.0 & 2.02 & & \\
\hline \multicolumn{7}{|c|}{ OM digestibility, $\%$} \\
\hline Control & $49.4^{\mathrm{a}}$ & $48.6^{\mathrm{a}}$ & $54.8^{b}$ & 1.88 & 0.75 & 0.04 \\
\hline Monensin & $50.4^{\mathrm{a}}$ & $50.9^{\mathrm{a}}$ & $53.0^{\mathrm{b}}$ & 1.76 & & \\
\hline \multicolumn{7}{|c|}{ NDF digestibility, $\%$} \\
\hline Control & $44.4^{\mathrm{a}}$ & $36.3^{b}$ & $49.2^{\mathrm{b}}$ & 2.61 & 0.67 & $<0.01$ \\
\hline Monensin & $44.7^{\mathrm{a}}$ & $39.8^{b}$ & $47.9^{b}$ & 2.44 & & \\
\hline \multicolumn{7}{|c|}{ ADF digestibility, $\%$} \\
\hline Control & $36.3^{\mathrm{a}}$ & $25.7^{b}$ & $43.5^{\mathrm{a}}$ & 3.69 & 0.79 & $<0.01$ \\
\hline Monensin & $36.9^{\mathrm{a}}$ & $29.9^{b}$ & $40.9^{a}$ & 3.45 & & \\
\hline
\end{tabular}

${ }^{a}$ No treatment $\times$ day interactions present $(P \geq 0.17)$.

${ }^{b, c, d}$ Corresponds with 71,94 , and $213 \mathrm{~d}$ of pregnancy, respectively.

${ }^{e}$ Pooled SE of least squares means ( $\operatorname{control} n=7$; monensin $n=8$ ).

a,b,c Within a row, means across days without a common superscript differ $(P \leq 0.05)$. 
BW tended to differ between $\mathrm{CON}$ and MON heifers (Table 4; $P=0.08$ ); however, when expressed as change in $\mathrm{BW}$ from initial weight on $\mathrm{d} 0$, no differences were detected between treatments $(P=0.32)$. Both BW and BW change were greater on $\mathrm{d} 161$ than all other days $(P<0.01)$.

Methane production was not different between treatments when expressed as daily liters of methane (Table 5; $P=0.40$ ). Additionally, methane production was least on $\mathrm{d} 0(P<0.02)$ and greatest on d $161(P<0.01)$. Nonetheless, MON heifers produced $7 \%$ less $(P=0.03)$ methane per day than $\mathrm{CON}$ heifers when expressed as liters of methane produced on a metabolic body weight (MBW) basis.

GE intake did not differ (Table 6; $P=0.91$ ) between treatments and increased from d 14 to d 161 to account for fetal growth requirements $(P<0.01)$. Fecal energy loss was not different between treatments when evaluated as megacalories (Mcal) $(P=0.91)$ or as a proportion of GE intake $(P=0.60)$, yet it did differ by day $(P<0.01)$. DE intake was not different between treatments when reported in Mcal $(P \geq 0.75)$ or as a proportion of GE intake $(P=0.60)$, but it was different across days $(P<0.01)$, being greater for $\mathrm{d} 161$ than $\mathrm{d} 14$ or d 42. Mcal of urinary energy loss did not differ across treatment $(P=0.28)$, neither did urinary energy loss as a proportion of GE intake $(P=0.26)$. However, Mcal of urine energy loss was greater on d 161 than on $\mathrm{d} 14$ or $\mathrm{d} 42(P<0.01)$. Methane energy loss in Mcal $(P=0.26)$ or as a proportion of GE intake $(P=0.78)$ was not different across treatment. Methane energy (in both Mcal and as a proportion of GE intake) was different across day $(P<0.01)$.

Mcal of ME intake was not different across treatment (Table 6; $P=0.58$ ), but was different across day $(P<0.01)$ being greatest on $\mathrm{d} 161$, least on d 14, and intermediate on d 42. Similarly, as a proportion of GE intake, ME intake was not different between CON and MON treatments $(P=0.42)$, where ME intake as a proportion of GE intake was

Table 4. BW measurements of drylot heifers receiving corn stalk-based diets with or without $150 \mathrm{mg} / \mathrm{d}$ of monensin at a maintenance level of intake

\begin{tabular}{|c|c|c|c|c|c|c|c|c|c|}
\hline \multirow[b]{2}{*}{ Item } & \multicolumn{6}{|c|}{ Day of study } & \multirow[b]{2}{*}{$\mathrm{SEM}^{b}$} & \multicolumn{2}{|c|}{ Probability $^{a}$} \\
\hline & 0 & 3 & 14 & 28 & 42 & 161 & & Treatment & Day \\
\hline \multicolumn{10}{|l|}{$\overline{\mathrm{BW}}, \mathrm{kg}$} \\
\hline Control & $472^{\mathrm{a}}$ & $469^{\mathrm{a}}$ & $448^{\mathrm{a}}$ & $461^{\mathrm{a}}$ & $457^{\mathrm{b}}$ & $525^{\mathrm{c}}$ & 10.9 & 0.08 & $<0.01$ \\
\hline Monensin & $494^{a}$ & $493^{\mathrm{a}}$ & $486^{\mathrm{a}}$ & $493^{\mathrm{a}}$ & $488^{\mathrm{a}}$ & $556^{\mathrm{b}}$ & 10.9 & & \\
\hline \multicolumn{10}{|c|}{ BW change, $\mathrm{kg}^{c}$} \\
\hline Control & $0^{\mathrm{a}}$ & $-3.4^{\mathrm{a}}$ & $-10.6^{\mathrm{a}}$ & $2.6^{\mathrm{a}}$ & $-3.6^{\mathrm{a}}$ & $68.4^{\mathrm{b}}$ & 3.78 & 0.32 & $<0.01$ \\
\hline Monensin & $0^{\mathrm{a}}$ & $-1.0^{\mathrm{a}}$ & $-6.8^{a}$ & $6.6^{\mathrm{a}}$ & $-4.1^{\mathrm{a}}$ & $67.9^{\mathrm{b}}$ & 3.57 & & \\
\hline
\end{tabular}

${ }^{a}$ No treatment $\times$ day interactions present $(P \geq 0.90)$.

${ }^{b}$ Pooled SE of least squares means (control $n=7$; monensin $n=8$ ).

${ }^{c} \mathrm{BW}$ change was calculated as the difference in BW from the previous weigh day.

${ }^{\mathrm{a}, \mathrm{b}, \mathrm{c}}$ Within a row, means across days without a common superscript differ $(P \leq 0.05)$.

Table 5. Daily methane production of heifers receiving corn stalk-based diets with or without $150 \mathrm{mg} / \mathrm{d}$ of monensin fed at a maintenance level of intake

\begin{tabular}{|c|c|c|c|c|c|c|c|c|c|}
\hline \multirow[b]{2}{*}{ Item } & \multicolumn{6}{|c|}{ Day of study } & \multirow[b]{2}{*}{$\mathrm{SEM}^{b}$} & \multicolumn{2}{|c|}{ Probability $^{a}$} \\
\hline & 0 & 3 & 14 & 28 & 42 & 161 & & Treatment & Day \\
\hline \multicolumn{10}{|l|}{$\overline{\mathrm{CH}_{4}, \mathrm{~L} / \mathrm{d}}$} \\
\hline Control & $134.4^{\mathrm{a}}$ & $160.8^{\mathrm{b}, \mathrm{c}}$ & $169.8^{c}$ & $158.3^{\mathrm{b}}$ & $161.6^{\mathrm{b}, \mathrm{c}}$ & $185.8^{\mathrm{d}}$ & 7.61 & 0.40 & $<0.01$ \\
\hline Monensin & $136.3^{\mathrm{a}}$ & $148.8^{\mathrm{b}, \mathrm{c}}$ & $159.3^{\mathrm{c}}$ & $146.8^{\mathrm{b}}$ & $163.7^{\mathrm{b}, \mathrm{c}}$ & $187.3^{\mathrm{d}}$ & 7.17 & & \\
\hline \multicolumn{10}{|c|}{$\mathrm{CH}_{4}, \mathrm{~L} / \mathrm{kg}$ of $\mathrm{DMI}$} \\
\hline Control & $26.0^{\mathrm{a}, \mathrm{c}}$ & $29.2^{\mathrm{b}}$ & $28.2^{\mathrm{a}, \mathrm{d}}$ & $26.4^{\mathrm{a}, \mathrm{c}}$ & $30.5^{\mathrm{b}, \mathrm{d}}$ & $22.62^{\mathrm{c}}$ & 1.72 & 0.51 & $<0.01$ \\
\hline Monensin & $23.5^{\mathrm{a}, \mathrm{c}}$ & $32.5^{\mathrm{b}}$ & $26.7^{\mathrm{a}, \mathrm{d}}$ & $23.4^{\mathrm{a}, \mathrm{c}}$ & $29.4^{\mathrm{b}, \mathrm{d}}$ & $23.3^{\mathrm{c}}$ & 1.72 & & \\
\hline \multicolumn{10}{|c|}{$\mathrm{CH}_{4}, \mathrm{~L} / \mathrm{kg} \mathrm{BW}^{0.75}$} \\
\hline Control & $1.3^{\mathrm{a}}$ & $1.6^{\mathrm{b}}$ & $1.7^{\mathrm{b}}$ & $1.5^{\mathrm{b}}$ & $1.6^{\mathrm{b}}$ & $1.7^{\mathrm{b}}$ & 0.07 & 0.03 & $<0.01$ \\
\hline Monensin & $1.3^{\mathrm{a}}$ & $1.4^{\mathrm{b}}$ & $1.5^{\mathrm{b}}$ & $1.4^{\mathrm{b}}$ & $1.5^{\mathrm{b}}$ & $1.6^{\mathrm{b}}$ & 0.06 & & \\
\hline
\end{tabular}

${ }^{a}$ No treatment $\times$ day interactions present $(P \geq 0.69)$.

${ }^{b}$ Pooled SE of least squares means (control $n=7$; monensin $n=8$ ).

a,b,c Within a row, means across days without a common superscript differ $(P \leq 0.05)$. 
Table 6. Daily energy partitioning of drylot heifers receiving corn stalk-based diets with or without $150 \mathrm{mg} / \mathrm{d}$ of monensin at a maintenance level of intake

\begin{tabular}{|c|c|c|c|c|c|c|}
\hline \multirow[b]{2}{*}{ Item } & \multicolumn{3}{|c|}{ Day of study } & \multirow[b]{2}{*}{$\mathrm{SEM}^{e}$} & \multicolumn{2}{|c|}{ 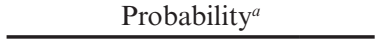 } \\
\hline & $14^{b}$ & $42^{c}$ & $161^{d}$ & & Treatment & Day \\
\hline \multicolumn{7}{|c|}{ GE intake, Mcal } \\
\hline Control & $23.80^{\mathrm{a}}$ & $20.71^{\mathrm{b}}$ & $32.54^{\circ}$ & 0.641 & 0.91 & $<0.01$ \\
\hline Monensin & $23.48^{\mathrm{a}}$ & $21.84^{\mathrm{b}}$ & $31.95^{\mathrm{c}}$ & 0.612 & & \\
\hline \multicolumn{7}{|c|}{ Fecal energy loss, Mcal } \\
\hline Control & $12.72^{\mathrm{a}}$ & $10.85^{\mathrm{b}}$ & $15.39^{\circ}$ & 0.523 & 0.91 & $<0.01$ \\
\hline Monensin & $12.52^{\mathrm{a}}$ & $10.87^{\mathrm{b}}$ & $15.43^{\circ}$ & 0.494 & & \\
\hline \multicolumn{7}{|c|}{ Fecal energy loss, $\%$ of GE intake } \\
\hline Control & $53.5^{\mathrm{a}}$ & $52.4^{\mathrm{a}}$ & $47.4^{\mathrm{b}}$ & 1.595 & 0.60 & $<0.01$ \\
\hline Monensin & $53.2^{\mathrm{a}}$ & $49.8^{\mathrm{a}}$ & $48.2^{\mathrm{b}}$ & 1.494 & & \\
\hline \multicolumn{7}{|c|}{ DE intake, Mcal } \\
\hline Control & $11.08^{\mathrm{a}}$ & $9.86^{\mathrm{a}}$ & $17.12^{\mathrm{b}}$ & 0.482 & 0.75 & $<0.01$ \\
\hline Monensin & $10.96^{\mathrm{a}}$ & $10.97^{\mathrm{a}}$ & $16.53^{b}$ & 0.490 & & \\
\hline \multicolumn{7}{|c|}{$\mathrm{DE}, \%$ of GE intake } \\
\hline Control & $46.52^{\mathrm{a}}$ & $47.63^{b}$ & $52.56^{\mathrm{b}}$ & 1.493 & 0.60 & $<0.01$ \\
\hline Monensin & $46.76^{\mathrm{a}}$ & $50.22^{\mathrm{b}}$ & $51.80^{\mathrm{b}}$ & 1.489 & & \\
\hline \multicolumn{7}{|c|}{ Urine energy, Mcal } \\
\hline Control & $1.11^{\mathrm{a}}$ & $0.97^{\mathrm{a}}$ & $1.32^{\mathrm{b}}$ & 0.069 & 0.28 & $<0.01$ \\
\hline Monensin & $1.05^{\mathrm{a}}$ & $0.88^{\mathrm{a}}$ & $1.33^{\mathrm{b}}$ & 0.069 & & \\
\hline \multicolumn{7}{|c|}{ Urine energy, $\%$ of GE intake } \\
\hline Control & 4.72 & 4.73 & 4.46 & 0.293 & 0.26 & 0.28 \\
\hline Monensin & 4.46 & 4.05 & 4.19 & 0.293 & & \\
\hline \multicolumn{7}{|c|}{$\mathrm{CH}_{4}$ energy, Mcal } \\
\hline Control & $1.61^{\mathrm{a}}$ & $1.53^{\mathrm{b}}$ & $1.76^{\mathrm{a}}$ & 0.082 & 0.78 & 0.01 \\
\hline Monensin & $1.51^{\mathrm{a}}$ & $1.55^{\mathrm{b}}$ & $1.77^{\mathrm{b}}$ & 0.082 & & \\
\hline \multicolumn{7}{|c|}{$\mathrm{CH}_{4}$ energy, $\%$ of GE intake } \\
\hline Control & $6.7^{\mathrm{a}}$ & $7.4^{\mathrm{b}}$ & $5.4^{\circ}$ & 0.302 & 0.57 & $<0.01$ \\
\hline Monensin & $6.4^{\mathrm{a}}$ & $7.1^{\mathrm{b}}$ & $5.5^{\mathrm{c}}$ & 0.281 & & \\
\hline \multicolumn{7}{|c|}{ ME intake, Mcal } \\
\hline Control & $8.36^{\mathrm{a}}$ & $7.36^{\mathrm{b}}$ & $14.03^{\mathrm{c}}$ & 0.474 & 0.58 & $<0.01$ \\
\hline Monensin & $8.41^{\mathrm{a}}$ & $8.55^{\mathrm{b}}$ & $13.42^{\circ}$ & 0.443 & & \\
\hline \multicolumn{7}{|c|}{$\mathrm{ME}, \%$ of GE intake } \\
\hline Control & $35.07^{\mathrm{a}}$ & $33.53^{\mathrm{a}}$ & $43.06^{\mathrm{b}}$ & 1.191 & 0.42 & $<0.01$ \\
\hline Monensin & $35.88^{\mathrm{a}}$ & $39.09^{\mathrm{b}}$ & $42.08^{b}$ & 1.684 & & \\
\hline \multicolumn{7}{|l|}{ ME:DE } \\
\hline Control & $0.75^{\mathrm{a}}$ & $0.74^{a}$ & $0.82^{\mathrm{b}}$ & 0.022 & 0.22 & $<0.01$ \\
\hline Monensin & $0.77^{\mathrm{a}}$ & $0.77^{\mathrm{a}}$ & $0.81^{\mathrm{b}}$ & 0.017 & & \\
\hline \multicolumn{7}{|l|}{ HP, Mcal } \\
\hline Control & $11.58^{\mathrm{a}}$ & $10.99^{\mathrm{b}}$ & $14.71^{\mathrm{c}}$ & 0.454 & 0.18 & $<0.01$ \\
\hline Monensin & $12.31^{\mathrm{a}}$ & $11.94^{\mathrm{b}}$ & $15.20^{\mathrm{c}}$ & 0.454 & & \\
\hline \multicolumn{7}{|c|}{$\mathrm{HP}, \%$ of GE intake } \\
\hline Control & $48.9^{\mathrm{a}}$ & $53.2^{\mathrm{b}}$ & $45.3^{\mathrm{c}}$ & 1.771 & 0.16 & $<0.01$ \\
\hline Monensin & $52.4^{\mathrm{a}}$ & $54.7^{\mathrm{b}}$ & $47.7^{\mathrm{c}}$ & 1.667 & & \\
\hline \multicolumn{7}{|l|}{ RE, Mcal } \\
\hline Control & $-3.22^{\mathrm{a}}$ & $-3.64^{a}$ & $-0.67^{b}$ & 0.696 & 0.36 & $<0.01$ \\
\hline Monensin & $-3.90^{\mathrm{a}}$ & $-3.40^{\mathrm{a}}$ & $-1.78^{b}$ & 0.651 & & \\
\hline \multicolumn{7}{|c|}{$\mathrm{RE}, \%$ of GE intake } \\
\hline Control & $-13.80^{\mathrm{a}}$ & $-17.70^{\mathrm{a}}$ & $-2.17^{b}$ & 2.635 & 0.56 & $<0.01$ \\
\hline Monensin & $-16.48^{a}$ & $-15.56^{\mathrm{a}}$ & $-5.63^{b}$ & 2.635 & & \\
\hline
\end{tabular}

${ }^{a}$ No treatment $\times$ day interactions present $(P \geq 0.12)$.

${ }^{b, c, d}$ Corresponds with 71,94 , and $213 \mathrm{~d}$ of pregnancy, respectively.

${ }^{e}$ Pooled SE of least squares means (control $n=7$; monensin $n=8$ ).

${ }^{\mathrm{a}, \mathrm{b}, \mathrm{c}}$ Within a row, means across days without a common superscript differ $(P \leq 0.05)$. 
greatest on d 161, than on d 14, with d 42 being intermediate $(P<0.01)$. The ME:DE was not affected by monensin inclusion $(P=0.22)$, but it did increase from d 14 and d 42 to $\mathrm{d} 161(P<0.01)$. Mcal of HP was not different between treatments $(P=0.16)$, and HP as a proportion of GE intake was not affected by monensin $(P=0.16)$, but was affected by day in that it was different on $\mathrm{d} 14$ than $\mathrm{d} 42$ than $\mathrm{d} 161(P<0.01)$. When adjusted for GE intake, HP followed the same trend where it was different $(P<0.01)$ on $\mathrm{d} 14$ than $\mathrm{d} 42$ than d 161, increasing each collection period. Retained energy in Mcal was not different across treatment $(P=0.36)$, but it was greater on $\mathrm{d} 14$ and $\mathrm{d} 42$ than d $161(P<0.01)$. Furthermore, retained energy as a proportion of GE intake did not differ when feeding monensin $(P=0.56)$, yet it differed across days, being greater on d 14 and d 42 than d $161(P$ $<0.01)$.

Nitrogen intake and excretion was not different between treatment groups (Table 7; $P \geq$ $0.13)$, and thus retained nitrogen for MON heifers was not different from CON heifers $(P=0.43)$. However, fecal and urinary nitrogen excretion as a percent of total nitrogen excretion was less for heifers fed monensin than control-fed heifers (Table $8 ; P<0.04$ ).

\section{DISCUSSION}

Limit-fed heifers in the present study did not differ in DMI due to monensin inclusion in the diet. Monensin is commonly reported to reduce DMI (Raun et al., 1976; Joyner et al., 1979; Byers, 1980; Goodrich et al., 1984), but typically this has been reported in ad libitum-fed cattle. The extent of the DMI response may be dependent on diet composition and concentration of monensin in the diet. However, the depression in DMI caused by monensin seems to be most noticeable in cattle receiving greater amounts of readily fermentable carbohydrates than forage-based diets (Bergen and Bates, 1984). Additionally, Raun et al. (1976) noted that DMI is not affected by monensin when dietary intake is restricted. Thus, no DMI differences were expected in the present experiment, and being fed near maintenance the heifers mostly consumed $100 \%$ of their daily feed allotment.

DM, OM, NDF, and ADF digestion did not differ when monensin was included in the diet. Likewise, when monensin was evaluated in situ, Dinius et al. (1976) noted no differences in DM or carbohydrate digestion with or without monensin. However, Wedegaertner and Johnson (1983) reported that monensin improved DM and NDF

Table 7. Nitrogen intake and excretion of drylot heifers receiving corn stalk-based diets with or without $150 \mathrm{mg} / \mathrm{d}$ of monensin at a maintenance level of intake

\begin{tabular}{|c|c|c|c|c|c|c|}
\hline \multirow[b]{2}{*}{ Item } & \multicolumn{3}{|c|}{ Day of study } & \multirow[b]{2}{*}{$\mathrm{SEM}^{e}$} & \multicolumn{2}{|c|}{ Probability $^{a}$} \\
\hline & $14^{b}$ & $42^{c}$ & $161^{d}$ & & Treatment & Day \\
\hline \multicolumn{7}{|l|}{$\mathrm{N}$ intake $\mathrm{g} / \mathrm{d}$} \\
\hline Control & $82.2^{\mathrm{a}}$ & $92.4^{\mathrm{b}}$ & $103.0^{\mathrm{b}}$ & 3.97 & 0.94 & $<0.01$ \\
\hline Monensin & $81.2^{\mathrm{a}}$ & $97.3^{\mathrm{b}}$ & $99.9^{\mathrm{b}}$ & 3.72 & & \\
\hline \multicolumn{7}{|l|}{$\mathrm{N}$ excretion, g/d } \\
\hline \multicolumn{7}{|l|}{ Feces } \\
\hline Control & $50.6^{\mathrm{a}}$ & $43.5^{\mathrm{a}}$ & $76.5^{\mathrm{b}}$ & 3.97 & 0.13 & $<0.01$ \\
\hline Monensin & $44.9^{a}$ & $40.1^{\mathrm{a}}$ & $71.5^{\mathrm{b}}$ & 3.71 & & \\
\hline \multicolumn{7}{|l|}{ Urine } \\
\hline Control & $30.4^{\mathrm{a}}$ & $34.7^{\mathrm{a}}$ & $45.1^{\mathrm{b}}$ & 2.80 & 0.16 & $<0.01$ \\
\hline Monensin & $33.5^{\mathrm{a}}$ & $33.5^{\mathrm{a}}$ & $50.6^{b}$ & 2.65 & & \\
\hline \multicolumn{7}{|l|}{ Total } \\
\hline Control & $81.0^{\mathrm{a}}$ & $78.3^{\mathrm{a}}$ & $121.8^{b}$ & 4.58 & 0.46 & $<0.01$ \\
\hline Monensin & $78.4^{\mathrm{a}}$ & $73.6^{\mathrm{a}}$ & $122.1^{\mathrm{b}}$ & 4.30 & & \\
\hline \multicolumn{7}{|c|}{ Apparent $\mathrm{N}$ digested, g/d } \\
\hline Control & $31.6^{\mathrm{a}}$ & $48.9^{b}$ & $25.9^{a}$ & 6.11 & 0.17 & $<0.01$ \\
\hline Monensin & $36.3^{\mathrm{a}}$ & $57.2^{\mathrm{b}}$ & $28.4^{\mathrm{a}}$ & 5.78 & & \\
\hline \multicolumn{7}{|l|}{$\mathrm{N}$ retained, $\mathrm{g} / \mathrm{d}$} \\
\hline Control & $1.2^{\mathrm{a}}$ & $14.2^{\mathrm{b}}$ & $-19.3^{\mathrm{c}}$ & 5.89 & 0.43 & $<0.01$ \\
\hline Monensin & $2.9^{\mathrm{a}}$ & $23.7^{\mathrm{b}}$ & $-22.2^{\mathrm{c}}$ & 5.59 & & \\
\hline
\end{tabular}

${ }^{a}$ No treatment $\times$ day interactions present $(P \geq 0.58)$.

${ }^{b, c, d}$ Corresponds with 71, 94, and $213 \mathrm{~d}$ of pregnancy, respectively.

${ }^{e}$ Pooled SE of least squares means (control $n=7$; monensin $n=8$ ).

a,b,c Within a row, means across days without a common superscript differ $(P \leq 0.05)$. 
digestion in cattle consuming a cracked corn and corn silage diet fed above maintenance.

There was a tendency for BW to increase with monensin inclusion in the present study; however, when $\mathrm{BW}$ was calculated as the change from BW on $\mathrm{d} 0$, the difference was no longer significant. Therefore, the changes observed in BW were not likely induced by feeding monensin, but rather differed enough on d 0 to cause a treatment effect, even though the $\mathrm{d} 0 \mathrm{BW}$ covariate was not significant.

Total liters of $\mathrm{CH}_{4}$ produced each day as well as liters of $\mathrm{CH}_{4} / \mathrm{kg} \mathrm{DM}$ intake were not different between treatments. This is in contrast to the work of others who have reported that feeding monensin decreases the amount of methane produced by $16 \%$ when fed to growing steers on a lower forage diet and 24\% when fed in a higher forage diet (Thornton and Owens, 1981). Additionally, the concentration of methane production in vivo (Joyner et al., 1979) and in vitro (Dinius et al., 1976) has also been reported to be decreased when feeding monensin. However, in Bos indicus and Bos taurus steers consuming ad libitum bermudagrass hay, there were no differences in $\mathrm{CH}_{4}$-producing activity when monensin was included either 0 or $200 \mathrm{mg} / \mathrm{d}$ (Bell et al., 2017a).
When expressed as liters per $\mathrm{kg}$ of $\mathrm{MBW}$ per day, methane production was reduced by $7 \%$ in heifers receiving monensin compared to their control counterparts. Additionally, Wedegaertner and Johnson (1983) observed a 26\% reduction in methane per $\mathrm{kg}$ MBW between monensin and control steers receiving a cracked corn and corn silage diet fed above maintenance. Furthermore, in an in vitro study, Dinius et al. (1976) proposed a ruminal adaptation to monensin after $9 \mathrm{~d}$ of feeding when methane concentrations for monensin-treated cultures were no longer different from controls. This work has been cited numerous times eluding to an adaptation of rumen microbiota to monensin. Bell et al. (2017a) made only one observation of in vitro methane production in cattle $42 \mathrm{~d}$ after feeding monensin and found a $15.8 \%$ reduction. When viewed in light of the confluence of methane production, rate for control- and monensin-fed steers within 4 $\mathrm{d}$ of withdrawing monensin (Bell et al., 2017b) suggests that the effect of monensin on methane production persisted through $42 \mathrm{~d}$ of inclusion in the diet. Although liters of methane production per $\mathrm{kg}$ of MBW for CON and MON heifers in the present study were numerically similar on $\mathrm{d} 42$ and $\mathrm{d}$ 161 , there is no evidence to suggest an adaptation

Table 8. Nitrogen intake and excretion of drylot heifers receiving corn stalk-based diets with or without $150 \mathrm{mg} / \mathrm{d}$ of monensin at a maintenance level of intake

\begin{tabular}{|c|c|c|c|c|c|c|}
\hline \multirow[b]{2}{*}{ Item } & \multicolumn{3}{|c|}{ Day of study } & \multirow[b]{2}{*}{$\mathrm{SEM}^{e}$} & \multicolumn{2}{|c|}{ Probability $^{a}$} \\
\hline & $14^{b}$ & $42^{c}$ & $161^{d}$ & & Treatment & Day \\
\hline \multicolumn{7}{|c|}{$\begin{array}{l}\mathrm{N} \text { excretion, } \% \text { of total } \mathrm{N} \text { excretion } \\
\text { Feces }\end{array}$} \\
\hline Control & 62.3 & 55.8 & 62.9 & 2.44 & 0.04 & 0.13 \\
\hline Monensin & 57.4 & 54.7 & 58.2 & 2.29 & & \\
\hline \multicolumn{7}{|l|}{ Urine } \\
\hline Control & 37.7 & 44.3 & 37.1 & 2.44 & 0.04 & 0.13 \\
\hline Monensin & 42.6 & 45.3 & 41.8 & 2.29 & & \\
\hline \multicolumn{7}{|c|}{$\mathrm{N}$ excretion, $\%$ of $\mathrm{N}$ intake } \\
\hline \multicolumn{7}{|l|}{ Feces } \\
\hline Control & $61.5^{\mathrm{a}}$ & $47.2^{\mathrm{b}}$ & $76.1^{\mathrm{c}}$ & 5.23 & 0.18 & $<0.01$ \\
\hline Monensin & $55.3^{\mathrm{a}}$ & $41.4^{b}$ & $75.1^{\mathrm{c}}$ & 4.95 & & \\
\hline \multicolumn{7}{|l|}{ Urine } \\
\hline Control & $37.2^{\mathrm{a}}$ & $37.7^{\mathrm{a}}$ & $44.1^{\mathrm{b}}$ & 2.83 & 0.15 & $<0.01$ \\
\hline Monensin & $41.3^{\mathrm{a}}$ & $34.7^{\mathrm{a}}$ & $51.4^{\mathrm{b}}$ & 2.66 & & \\
\hline \multicolumn{7}{|c|}{$\mathrm{N}$ digested, $\%$ of $\mathrm{N}$ intake } \\
\hline Control & $38.5^{\mathrm{a}}$ & $52.8^{\mathrm{b}}$ & $23.9^{\mathrm{c}}$ & 4.88 & 0.09 & $<0.01$ \\
\hline Monensin & $44.7^{\mathrm{a}}$ & $58.6^{\mathrm{b}}$ & $27.3^{\mathrm{c}}$ & 4.62 & & \\
\hline \multicolumn{7}{|c|}{$\mathrm{N}$ retained, $\%$ of $\mathrm{N}$ intake } \\
\hline Control & $6.2^{\mathrm{a}}$ & $16.1^{\mathrm{b}}$ & $28.2^{\mathrm{c}}$ & 4.82 & 0.50 & $<0.01$ \\
\hline Monensin & $6.6^{\mathrm{a}}$ & $24.0^{\mathrm{b}}$ & $29.5^{\mathrm{c}}$ & 4.54 & & \\
\hline
\end{tabular}

${ }^{a}$ No treatment $\times$ day interactions present $(P \geq 0.25)$.

${ }_{b, c, d}$ Corresponds with 71,94 , and $213 \mathrm{~d}$ of pregnancy, respectively.

${ }^{e}$ Pooled SE of least squares means (control $n=7$; monensin $n=8$ ).

a,b,c Within a row, means across days without a common superscript differ $(P \leq 0.05)$. 
to monensin, as there was no treatment $\times$ day interaction.

GE intake was not different between treatment groups, and no differences were observed in DE or ME intake. In a study with lambs, Joyner et al. (1979) reported a 2.8\% increase in DE and an 8.1\% increase in $\mathrm{ME}$ when including monensin at $20 \mathrm{ppm}$ in the diet. Fecal, $\mathrm{CH}_{4}$, and urinary energy losses as a percent of GE intake were not different between treatments, which supports the lack of differences observed in DE and ME intakes. No differences in $\mathrm{HP}$ were noted for $\mathrm{CON}$ and MON heifers, and ultimately, RE did not change because of monensin and was slightly negative for both treatment groups throughout the experiment. Although Joyner et al. (1979) reported a $15 \%$ increase in RE when feeding monensin, the decrease in methane production in the present study was small, and thus it was not substantial enough to alter RE. Boardman (2015) also observed no change in HP or RE when monensin was included at $200 \mathrm{mg} / \mathrm{d}$ in beef cow diets fed at $80 \%$ or $120 \%$ of NRC (2000) requirements. Likewise, Thornton and Owens (1981) noted no difference in urinary energy loss, ME values, or HP by feeding monensin. Nonetheless, when averaged across three concentrations of forage inclusion (low, medium, and high), the inclusion of $200 \mathrm{mg} / \mathrm{d}$ of monensin did increase the ME of the diets by $5.2 \%$. It was concluded that the increase in $\mathrm{ME}$ was because of decreased $\mathrm{CH}_{4}$ energy loss and the tendency for monensin to decrease fecal energy loss.

The ME:DE ratio observed in the current study ranged from 0.74 to 0.82 . The NRC (2000) suggested that for most forages and mixtures of forages and cereal grains the ratio of $\mathrm{ME}$ to $\mathrm{DE}$ is about 0.8 , which is consistent with our data. Furthermore, we used the equation for predicting $\mathrm{ME}(\mathrm{ME}=0.96 \times$ $\mathrm{DE}-0.30)$ that was developed by Galyean et al. (2016) and determined the values were similar to the actual ME observed in the current experiment.

Both CON and MON heifers maintained their BW through d 42 of feeding monensin and had a large increase in BW from d 42 to $\mathrm{d} 161$ despite having a negative energy balance for the duration of the trial. Day 161 corresponded to approximately d 210 of gestation when rapid fetal growth takes place, which may account for some of the observed increase in BW, as very little energy and nitrogen are stored in the gravid uterine tissues early in gestation relative to the amounts stored during late gestation (Ferrell et al., 1976). When conceptus and tissue weights were predicted using the NRC (2000) model and were subtracted from heifer BW, $\mathrm{BW}$ gain remained positive after accounting for changes due to fetal growth. This is perplexing, as the RE was negative on d 161, but the negative RE was likely used to maintain pregnancy. Using equations described by Ferrell et al. (1976) based on days pregnant and predicted calf birth weight, the cows required a range of 0.5 and $1.2 \mathrm{Mcal} / \mathrm{d}$ to maintain pregnancy throughout the study. The energy required for the gravid uterus could partially explain why the heifers were in a negative energy balance during each collection. Other potential explanations include differences in intake during collection periods, effects of limit feeding, or a combination of these factors. For instance, DMI during the collection periods may have been lower than intake between collections, which would have caused RE to be negative just for the days on which $\mathrm{RE}$ was measured. The heifers across both treatments consumed approximately 8\% less DM during the last collection period than the $3 \mathrm{wk}$ prior in the Calan gate bunks which is likely because DMI usually decreases in late pregnancy. Additionally, the last collection period occurred during late April and the ambient temperature (mean high temperature $=17.7^{\circ} \mathrm{C}$, mean low temperature $=4.5^{\circ} \mathrm{C}$ ) was greater than the collection periods that occurred in November (mean high temperature $=9.3{ }^{\circ} \mathrm{C}$, mean low temperature $=-1.4{ }^{\circ} \mathrm{C}$ ) and December (mean high temperature $=5.6{ }^{\circ} \mathrm{C}$, mean low temperature $=-4.2{ }^{\circ} \mathrm{C}$ ) and potentially the heifers consumed more water, possibly causing an increase in gut fill before BW was collected. The increased ambient temperature during the April collection (d 161) could have also contributed to the decrease in DMI during the collection period. Previous limit-feeding trials (Trubenbach, 2014; Boardman, 2015; Baber et al., 2016) have observed increases in $\mathrm{BW}$ following a period of nutrient restriction, which is hypothesized to be a result of metabolic adaptation to limited energy intake.

Nitrogen consumption for $\mathrm{CON}$ and $\mathrm{MON}$ heifers was not different. Fecal and urinary nitrogen excretion also did not differ between treatments so that retained nitrogen was not affected by monensin inclusion. This response was not expected, as monensin is known for having a protein sparing effect in the rumen, leading to greater protein absorption in the small intestine and increased nitrogen retention (Russell and Strobel, 1989). Joyner et al. (1979) reported improved nitrogen digestion with monensin additionally urinary nitrogen excretion was reduced and retained nitrogen was increased. Likewise, Dinius et al. (1976) observed a tendency for monensin-fed steers to have greater nitrogen retention than control-fed steers. 
Results of this experiment indicate that adding monensin to limit-fed, corn stalk-based diets may not have a large effect on the energy and nitrogen balance of confined heifers. The failure to detect differences may be related to low statistical power and only using 15 heifers. Moreover, DMI and nutrient digestion were not affected by monensin. Methane production per $\mathrm{kg} \mathrm{MBW}$ was decreased by $7 \%$ in $\mathrm{MON}$ heifers compared to $\mathrm{CON}$ heifers. Furthermore, fecal, $\mathrm{CH}_{4}$, urinary, and heat energy losses were not reduced by monensin, as has been reported previously, thus no changes were observed in DE and ME intake or RE. Many of the energy saving effects of monensin were not observed in this study, and that could be a result of feeding monensin at a lower level $(150 \mathrm{mg} / \mathrm{d})$ rather than a higher level that was often fed in the other studies that reported differences. Lack of an energy saving effect by monensin could be a result of the heifers being fed near maintenance rather than ad libitum. Further research should be conducted to determine the efficacy of including monensin in limit-fed, high-forage diets in drylot cow systems.

\section{ACKNOWLEDGMENTS}

Mention of trade names or commercial products in this article is solely for the purpose of providing specific information and does not imply recommendation or endorsement by the USDA. USDA is an equal opportunity provider and employer. The efforts of A. Menke, L. Hassler, and C. Engle in the conduct of this research and the cattle management are greatly appreciated.

\section{Conflict of interest statement. None declared.}

\section{LITERATURE CITED}

Baber, J. R., J. E. Sawyer, L. A. Trubenbach, and T. A. Wickersham. 2016. Effect of time of concentrate delivery on nutrient digestibility, ruminal fermentation, and solid passage rate in limit-fed steers consuming wheat straw. J. Anim. Sci. 94(E-Suppl. 1):43. doi:10.2527/ ssasas2015-031

Bell, N. L., R. C. Anderson, T. R. Callaway, M. O. Franco, J. E. Sawyer, and T. A. Wickersham. 2017a. Effect of monensin inclusion on intake, digestion, and ruminal fermentation parameters by Bos taurus indicus and Bos taurus steers consuming bermudagrass hay. J. Anim. Sci. 95:2736-2746. doi:10.2527/jas2016.1013

Bell, N. L., R. C. Anderson, T. R. Callaway, M. O. Franco, J. E. Sawyer, and T. A. Wickersham. 2017b. Effect of monensin withdraw on intake, digestion, and ruminal fermentation parameters by Bos taurus indicus and Bos taurus steers consuming bermudagrass hay. J. Anim. Sci. 95:2747-2757. doi:10.2527/jas2016.1011
Bergen, W. G., and D. B. Bates. 1984. Ionophores: their effect on production efficiency and mode of action. J. Anim. Sci. 58:1465-1483. doi:10.2527/jas1984.5861465x

Boardman, C. J. 2015. Effects of monensin and dietary energy intake on maintenance requirements in beef cows [M.S. thesis]. College Station (TX): Texas A\&M University.

Brouwer, E. 1965. Report of sub-committee on constants and factors. In: Energy metabolism, EAAP Publ. No. 11, Troon, UK; p. 441.

Byers, F. M. 1980. Determining effects of monensin on energy value of corn silage diets for beef cattle by linear or semi-log methods. J. Anim. Sci. 51:158-169. doi:10.2527/ jas1980.511158x

Dinius, D. A., M. E. Simpson, and P. B. Marsh. 1976. Effect of monensin fed with forage on digestion and the ruminal ecosystem of steers. J. Anim. Sci. 42:229-234. doi:10.2527/ jas1976.421229x

Duffield, T. F., J. K. Merrill, and R. N. Bagg. 2012. Metaanalysis of the effects of monensin in beef cattle on feed efficiency, body weight gain, and dry matter intake. J. Anim. Sci. 90:4583-4592. doi:10.2527/jas.2011-5018

Eng, K. S. 2014. Nutritional and management considerations when merging cow-calf and feedlot operations. In: Dr. Kenneth S. and Caroline McDonald Eng Foundation Symposium, San Antonio, TX; p. 43.

Ferrell, C. L., W. N. Garrett, and N. Hinman. 1976. Growth, development and composition of the udder and gravid uterus of beef heifers during pregnancy. J. Anim. Sci. 42:1477-1489. doi:10.2527/jas1976.4261477x

Freetly, H. C., and J. A. Nienaber. 1998. Efficiency of energy and nitrogen loss and gain in mature cows. J. Anim. Sci. 78:896-905. doi:10.2527/1998.763896x

Galyean, M. L., N. A. Cole, L. O. Tedeschi, and M. E. Branine. 2016. BOARD-INVITED REVIEW: efficiency of converting digestible energy to metabolizable energy and reevaluation of the California Net Energy System maintenance requirements and equations for predicting dietary net energy values for beef cattle. J. Anim. Sci. 94:1329 1341. doi: $10.2527 /$ jas.2015-0223

Goodrich, R. D., J. E. Garrett, D. R. Gast, M. A. Kirick, D. A. Larson, and J. C. Meiske. 1984. Influence of monensin on the performance of cattle. J. Anim. Sci. 58:1484-1498. doi: $10.2527 /$ jas $1984.5861484 x$

Joyner, A. E. Jr., L. J. Brown, T. J. Fogg, and R. T. Rossi. 1979. Effect of monensin on growth, feed efficiency and energy metabolism of lambs. J. Anim. Sci. 48:1065-1069. doi:10.2527/jas1979.4851065x

Nienaber, J. A., and A. L. Maddy. 1985. Temperature controlled multiple chamber indirect calorimeter-design and operation. Trans. ASAE 28:555-560. doi:10.13031/2013.32297

NRC. 2000. Nutrient requirements of beef cattle. 7 th rev. ed. Update. Washington (DC): National Academy Press.

Potter, E. L., C. O. Cooley, L. F. Richardson, A. P. Raun, and R. P. Rathmacher. 1976. Effect of monensin on performance of cattle fed forage. J. Anim. Sci. 43:665-669. doi:10.2527/jas1976.433665x

Raun, A. P., C. O. Cooley, E. L. Potter, R. P. Rathmacher, and L. F. Richardson. 1976. Effect of monensin on feed efficiency of feedlot cattle. J. Anim. Sci. 43:670-677. doi:10.2527/jas1976.433670x

Richardson, L. F., A. P. Raun, E. L. Potter, C. O. Cooley, and R. P. Rathmacher. 1976. Effect of monensin on rumen fermentation in vitro and in vivo. J. Anim. Sci. 43:657-664. doi: $10.2527 /$ jas $1976.433657 \mathrm{x}$ 
Russell, J. B., and H. J. Strobel. 1989. Effect of ionophores on ruminal fermentation. Appl. Environ. Microbiol. 55:1-6.

Thornton, J. H., and F. N. Owens. 1981. Monensin supplementation and in vivo methane production by steers. J. Anim. Sci. 52:628-634. doi:10.2527/jas1981.523628x

Tolleson, D. R., and L. L. Erlinger. 1989. An improved harness for securing fecal collection bags to grazing cattle. J. Range Manag. 45:396-399.
Trubenbach, L. A. 2014. Effects of energy density and intake on maintenance requirements in beef cows [M.S. thesis]. College Station (TX): Texas A\&M University.

Wedegaertner, T. C., and D. E. Johnson. 1983. Monensin effects on digestibility, methanogenesis and heat increment of a cracked corn-silage diet fed to steers. J. Anim. Sci. 57:168-177. doi:10.2527/jas1983.571168x 